\title{
Assessment of Clinical Symptoms in Lumbar Foraminal Stenosis Using the Japanese Orthopaedic Association Back Pain Evaluation Questionnaire
}

\begin{tabular}{|c|}
\hline 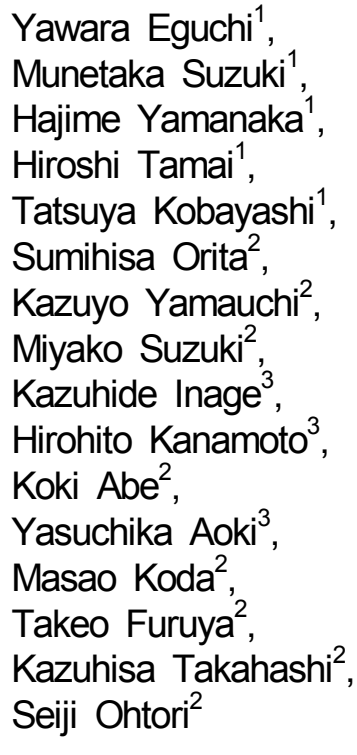 \\
\hline
\end{tabular}

${ }^{1}$ Department of Orthopedic Surgery, National Hospital Organization Shimoshizu National Hospital, Yotsukaido, ${ }^{2}$ Department of Orthopedic Surgery, Graduate School of Medicine, Chiba University, Chiba, ${ }^{3}$ Department of Orthopaedic Surgery, Eastern Chiba Medical Center, Togane, Chiba, Japan

\section{Corresponding Author:}

Yawara Eguchi

Department of Orthopaedic Surgery, Shimoshizu National Hospital, 934-5, Shikawatashi, Yotsukaido, Chiba 284-0003, Japan

Tel: +81-43-422-2511

Fax: +81-43-421-3007

E-mail: yawara_eguchi@yahoo.co.jp

Received: September 16, 2016

Revised: January 4, 2017

Accepted: February 1, 2017

Copyright (C) 2017 by The Korean Spinal Neurosurgery Society

This is an open access article distributed under the terms of the Creative Commons Attribution Non-Commercial License (http://creativecommons.org/licenses/by-n $\mathrm{c} / 4.0 /$ ) which permits unrestricted non-commercial use, distribution, and reproduction in any medium, provided the original work is properly cited.

Objective: It is important to develop an easy means of diagnosing lumbar foraminal stenosis (LFS) in a general practice setting. We investigated the use of the Japanese Orthopaedic Association Back Pain Evaluation Questionnaire (JOABPEQ) to diagnose LFS in symptomatic patients.

Methods: Subjects included 13 cases (mean age, 72 years) with LFS, and 30 cases (mean age, 73 years) with lumbar spinal canal stenosis (LSCS) involving one intervertebral disc. The visual analogue scale score for low back pain and leg pain, the JOABPEQ were evaluated. Results: Those with LFS had a significantly lower JOA score $(p<0.001)$, while JOABPEQ scores $(p<0.05)$ for lumbar dysfunction and social functioning impairment $(p<0.01)$ were both significantly lower than the scores in LSCS. The following JOABPEQ questionnaire items (LFS vs. LSCS, p-value) for difficulties in: sleeping $(53.8 \%$ vs. $16.6 \%, p<0.05)$, getting up from a chair $(53.8 \%$ vs. $6.6 \%, p<0.001)$, turning over $(76.9 \%$ vs. $40 \%, p<0.05)$, and putting on socks $(76.9 \%$ vs. $26.6 \%, p<0.01)$ such as pain during rest, and signs of intermittent claudication more than 15 minutes $(61.5 \%$ vs. $26.6 \%, p<0.05)$ were all significantly more common with LFS than LSCS. Conclusion: Results suggest that of the items in the JOABPEQ, if pain during rest or intermittent claudication is noted, LFS should be kept in mind as a cause during subsequent diagnosis and treatment. LFS may be easily diagnosed from LSCS using this established patient-based assessment method.

Key Words: Lumbar foraminal stenosis, Clinical symptom, Diagnosis, Japanese Orthopaedic Association Back Pain Evaluation Questionnaire

\section{INTRODUCTION}

Lumbar foraminal stenosis (LFS) is a condition seen in degenerative lumbar spinal disorders in which a nerve root or spinal nerve is entrapped in a narrowed lumbar foramen. There is a dorsal root ganglion that functions as a pain receptor at this site making this condition refractory and likely to cause severe lower limb pain ${ }^{10)}$. However, Macnab ${ }^{14)}$ suitably referred to this region as the "hidden zone" and despite major strides in imaging technology today, this site is still often overlooked, making it a factor that can adversely impact surgical success rates.

Nerve decompression sites differ in intraspinal lesions and foraminal stenosis, and it has been reported that many cases of failed back surgery syndrome are caused by inappropriate treatment of foraminal stenosis ${ }^{4}$. Conditions that cause L5 radiculopathy include a "doublecrush lesion" where the L4/5 level is compre- ssed by an intraspinal canal lesion and the L5/ S1 level is compressed by a lateral lesion so that the nerve is compressed at 2 points (medial and lateral), hence the name. However, traditional imaging studies do not allow the clinician to differentially diagnose whether the compressing lesion is inside or outside the spinal canal, or if a double-crush lesion is responsible.

The Japanese Orthopaedic Association Back Pain Evaluation Questionnaire (JOABPEQ) ${ }^{6}$ provides specific, yet multidimensional outcome measures for patients with low back pain (LBP), including dysfunction and disabilities caused by the disease, as well as resulting psychosocial problems. The reliability and validity of the JOABPEQ have been verified by psychometric evaluations.

It is important to develop an easy means of diagnosing LFS from LSCS. The purpose of this study was to investigate the use of the JOABPEQ to diagnose LFS in symptomatic patients. Our findings from using the JOABPEQ to study clinical symptoms in detail to determine the scale's 
usefulness in diagnosing LFS are presented below.

\section{MATERIALS AND METHODS}

Thirteen cases (mean age, 72 years) of LFS and 30 cases (mean age, 73 years) of LSCS involving one intervetebral space were enrolled as subjects from among the 143 patients (mean age, 66.8 years) who underwent lumbar surgery between April 2013 and October 2015 at our institution. LFS neuropathy was level L3 in 2 cases, L4 in 2 cases and L5 in 9 cases. LSCS was level

Table 1. Japanese Orthopaedic Association Back Pain Evaluation Questionnaire

Q1-1 To alleviate low back pain, you often change your posture.
1) Yes
2) No

Q1-2 Because of the low back pain, you lie down more often than usual.

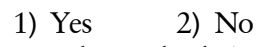

Q1-3 Your lower back is almost always aching.

$\begin{array}{ll}\text { 1) Yes } & \text { 2) No }\end{array}$

Q1-4 Be cause of the low back pain, you cannot sleep well.

1) No 2) Yes

Q2-1 Because of the low back pain, you sometimes ask someone to help you when you do something.
1) Yes
2) No

Q2-2 Because of the low back pain, you refrain from bending forward or kneeling down.

1) Yes 2) No

Q2-3 Because of the low back pain, you have difficulty standing up from a chair.

$\begin{array}{ll}\text { 1) Yes } & \text { 2) No }\end{array}$

Q2-4 Because of the low back pain, turning over in bed is difficult.
1) Yes
2) No

Q2-5 Because of the low back pain, you have difficulty putting on socks or stockings.
1) Yes
2) No

Q2-6 Do you have difficulty with any one of the following motions; bending forward, kneeling or stooping?
1) I have great difficulty.
2) I have some difficulty.
3) I have no difficulty.

Q3-1 Because of the low back pain, you walk only short distances.
1) Yes
2) No

Q3-2 Because of the low back pain, you stay seated most of the day.
1) Yes
2) No

Q3-3 Because of the low back pain, you go up the stairs more slowly than usual.
1) Yes
2) No

Q3-4 Do you have difficulty going up the stairs?
1) I have great difficulty.
2) I have some difficulty.
3) I have no difficulty.

Q3-5 Do you have difficulty walking more than 15 minutes?
1) I have great difficulty.
2) I have some difficulty.
3) I have no difficulty.

Q4-1 Because of the low back pain, you do not do any routine housework these days.
1) No
2) Yes

Q4-2 Have you been unable to do your work or ordinary activities as well as you would like?

1) I have not been able to do them at all.

2) I have been unable to do them most of the time.

3) I have sometimes been unable to do them.

4) I have been able to do them most of the time.

5) I have always been able to do them.

Q4-3 Has your work routine been hindered because of the pain?
1) Greatly
2) Moderately
3) Slightly (somewhat)
4) Little (minimally)
5) Not at all

Q5-1 Because of the low back pain, you get irritated or get angry at other persons more often than usual.
1) Yes
2) No

Q5-2 How is your present health condition?
1) Poor
2) Fair
3) Good
4) Very good
5) Excellent

Q5-3 Have you been discouraged and depressed?
1) Always
2) Frequently
3) Sometimes
4) Rarely
5) Never

Q5-4 Do you feel exhausted?
1) Always
2) Frequently
3) Sometimes
4) Rarely
5) Never

Q5-5 Have you felt happy?
1) Never
2) Rarely
3) Sometimes
4) Almost always
5) Always

Q5-6 Do you think you are in decent health?
1) Not at all 2) Barely
3) Not very much
4) Fairly
5) Yes

Q5-7 Do you feel your health will get worse?
1) Very much so
2) A little bit at a time
3) Sometimes yes and sometimes no
4) Not very much
5) Not at all 
$\mathrm{L} 1 / 2$ in 1 case, L2/3 in 2 cases, L3/4 in 5 cases, and L4/5 in 22 cases. LFS was diagnosed by microendoscopic intrapedicular partial pediculotomy in 3 cases $^{15)}$, and posterior lumbar interbody fusion in 10 cases. LSCS was diagnosed in all patients through lumbar spinous process-splitting laminectomy ${ }^{17}$. Before surgery, LFS was diagnosed comprehensively based on clinical symptoms, physical findings, plain X-rays, computed tomography $(\mathrm{CT})$, magnetic resonance imaging (MRI), and 3-dementional-MRI (3D-MRI). Foraminal stenosis was defined as: abnormalities such as nerve indentation, swelling, and running transversely in their course through the foramen on 3D-MRI. Ultimately, nerve roots were blocked selectively to diagnose damaged nerve roots based on function. If the visual analog scale (VAS $100-\mathrm{mm}$ method) of the lower limbs was alleviated by $20 \mathrm{~mm}$ or less at 30 minutes after nerve root block, the diagnosis was considered positive.

Patient exclusion criteria were as follows: (1) those with residual lower limb pain, (2) those who had previous lumbar spinal surgery, (3) those who had multiple levels of lumbar canal stenosis, (4) those who had myelopathy, and (5) those who had spinal tumor, infectious disease, or spinal trauma.

Clinical symptoms were evaluated using the VAS score for LBP and leg pain ranging from $100 \mathrm{~mm}$ (extreme amount of pain) to $0 \mathrm{~mm}$ (no pain), the Japanese Orthopedic Association (JOA; 0-29 points) scoring system, and the Roland-Morris Disability Questionnaire (RDQ; 0-24 points). The normal JOA score is 29 points, based on 3 subjective symptoms ( 9 points), 3 clinical signs including straight-leg raising (6 points), and 7 activities of daily living (14 points). The normal RDQ is zero points with the total number of items checked from a minimum of 0 to a maximum of 24 .

The JOABPEQ includes 25 questions based on RDQs and Short Form 36 (SF-36).

For Q1-1 through Q4-1 and Q5-1, a score of "1" was considered positive for symptoms, while a "2" or " 3 " was considered negative. For Q4-2, Q4-3, and Q5-2 through Q5-7, a score of "1" or " 2 " was considered positive for symptoms, and " 3 " to " 5 " were negative (Table 1). Scores are calculated based on the answers to questions in 5 domains: pain-related disorders, lumbar spine dysfunction, gait disturbance, social life dysfunction, and psychological disorders. The score for each domain was calculated according to the official guidelines and ranged from 0 to 100 points, which is deemed proportional to the patient's clinical condition.

All human and animal studies have been approved by the Chiba University and Shimoshizu National Hospital and have therefore been performed in accordance with the ethical standards laid down in the 1964 Declaration of Helsinki and its later amendments. We declare that all patients gave informed consent prior to inclusion in this study.

Statistical analyses were performed with StatView ver. 5.0 (SAS Institute Inc., Cary, NC, USA). For each clinical symptom, differences between both groups were evaluated using an unpaired t-test. For each JOABPEQ item, differences between both groups were evaluated using the chi-squared test. A threshold of $p<0.05$ was considered statistically significant.

\section{RESULTS}

VAS scores (LBP) were LFS: $73 \pm 5.17 \mathrm{~mm}$, LSCS: $60 \pm 4.67$ $\mathrm{mm}$ ( $\mathrm{p}=0.105)$; VAS scores (leg pain) were LFS: $80 \pm 5.37 \mathrm{~mm}$, LSCS: $65 \pm 5.25 \mathrm{~mm}(\mathrm{p}=0.090)$; JOA scores were LFS: $15 \pm 0.69$, LSCS: $19 \pm 0.54(\mathrm{p}=0.00047)$; RDQ scores were LFS: $13 \pm 1.30$, and LSCS: $11 \pm 0.99(\mathrm{p}=0.157)$. JOA scores were significantly lower $(\mathrm{p}<0.001)$ in LFS compared to LSCS (Fig. 1).

Categories in JOABPEQ include Pain-related disorders LFS: 38 \pm 7.48 , LSCS: $57 \pm 6.51(\mathrm{p}=0.087)$, Lumbar spine dysfunction LFS: $44 \pm 6.86$, LSCS: $67 \pm 4.22(\mathrm{p}=0.026)$, Gait disturbance LFS: 28 \pm 6.34 , LSCS: $41 \pm 5.01(\mathrm{p}=0.082)$, Social life disturbance LFS: $32 \pm 5.86$, LSCS: $47 \pm 3.28(\mathrm{p}=0.009)$, and Psychological disorders LFS: $35 \pm 5.81$, LSCS: $43 \pm 3.33(\mathrm{p}=0.199)$. LFS showed significantly lower scores in Lumbar dysfunction $(\mathrm{p}<0.05)$, and Social life disturbance $(\mathrm{p}<0.01)$ compared to LSCS (Fig. 2).

Statistically significant differences were noted between the 2 groups. Specifically, in Pain-related disorders. (1) have difficulty sleeping: LFS, $53.8 \%$; LSCS, $16.6 \%(\mathrm{p}=0.0125)$; in Lumbar spine dysfunction: (2) have difficulty standing up from a chair: LFS, $53.8 \%$; LSCS, $6.6 \%(\mathrm{p}=0.0004)$; (3) have difficulty turning over: LFS, 76.9\%; LSCS, 40\% ( $\mathrm{p}=0.0261)$; (4) have difficulty putting on socks or stockings: LFS, $76.9 \%$; LSCS, $26.6 \%(\mathrm{p}=0.0021)$; in Gait disturbances: (5) have difficulty walking more than 15 minutes: LFS, 61.5\%; LSCS, 26.6\% ( $\mathrm{p}=0.0298)$; in Social life disturbance: (6) do not do routine housework: LFS, 38.4\%; LSCS, $0 \%(\mathrm{p}=0.0003)$; and in Psychological disorders: (7) are not in decent health: LFS, 69.2\%; LSCS, 30.0\% ( $\mathrm{p}=0.0166)$. There were 7 question items and incidence was consistently higher in LFS

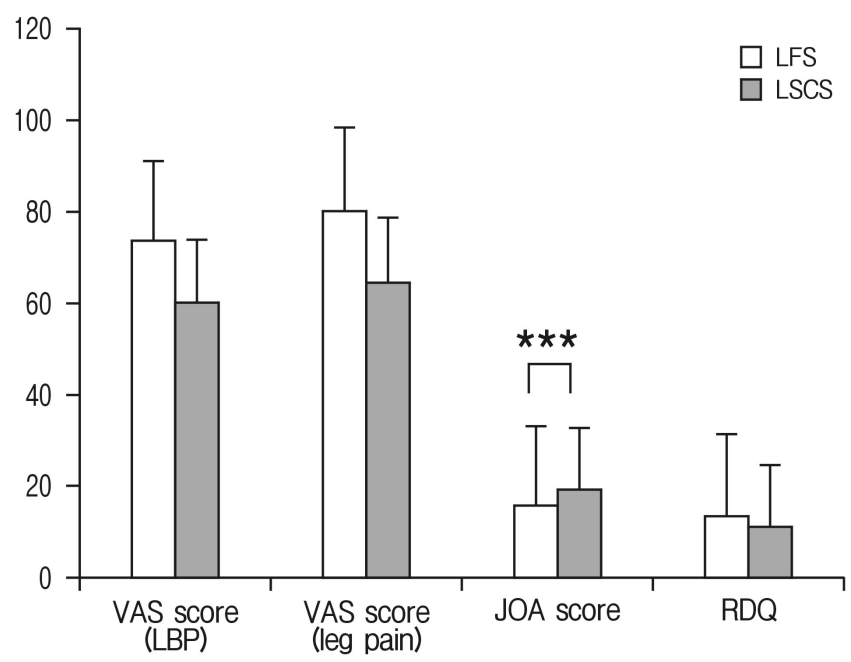

Fig. 1. VAS score, JOA score, RDQ in each group. Compared to LSCS, JOA scores were significantly lower $(p<0.001)$ in the LFS patients. VAS, visual analogue scale; JOA, Japanese Orthopaedic Association; RDQ, Roland-Morris Disability Questionnaire; LSCS, lumbar spinal canal stenosis; LFS, lumbar foraminal stenosis. $* * \mathrm{p}<0.001$. 


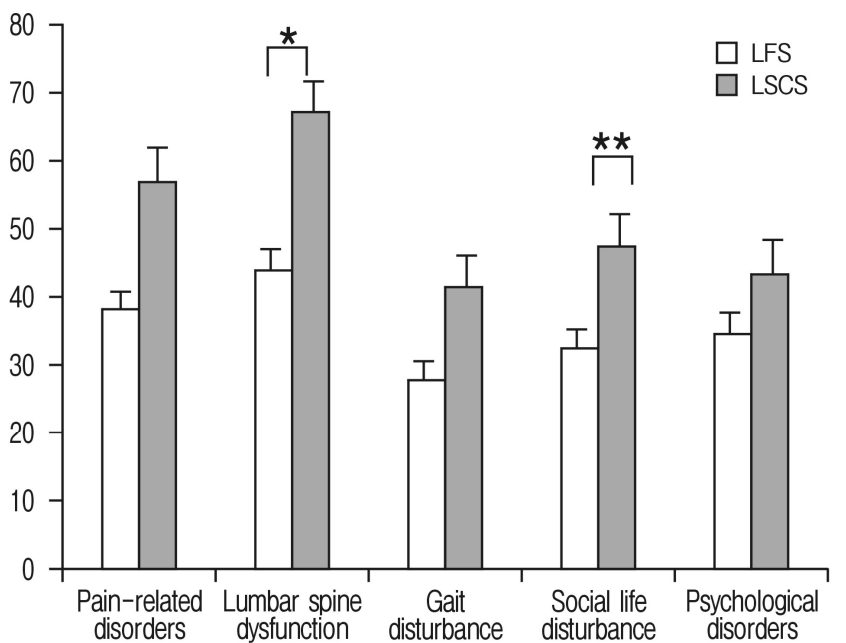

Fig. 2. JOABPEQ scores in each group. Compared to LSCS, LFS patients had significantly lower lumbar spine dysfunction $(\mathrm{p}<0.05)$ and social life disturbance $(\mathrm{p}<0.01)$ scores. JOABPEQ, Japanese Orthopaedic Association Back Pain Evaluation Questionnaire; LSCS, lumbar spinal canal stenosis; LFS, lumbar foraminal stenosis. *p $<0.05$. * $\mathrm{p}<0.01$. than LSCS (Table 2).

\section{DISCUSSION}

Appropriately named the "hidden zone" by Macnab ${ }^{14)}$, LFS is often overlooked, accounts for approximately $60 \%$ of failed back surgery syndromes, and plays a major role in lowering surgical success rates ${ }^{4)}$. Diagnostic imaging of lumbar spinal canal stenosis involves a comprehensive review of X-rays, CT, and $\mathrm{MRI}^{7,12,16)}$, together with functional diagnosis through selective nerve root imaging and infiltration ${ }^{8)}$. Conventional MRI reportedly produces false positives in $30 \%$ to $40 \%$ of LFS cases and this is therefore a difficult condition to diagnose. Recently, 3D-CT, MR myelography ${ }^{13)}$, 3D-MRI ${ }^{2,20)}$, and diffusion tensor imaging ${ }^{5)}$ have been reported to be diagnostically useful.

Nerve root damage in LFS is most common in the L5 nerve root, accounting for $75 \%$ of cases ${ }^{10)}$. There are no useful diagnostic methods to differentiate between possible causes of L5 nerve damage that could be medial stenotic lesions in the $\mathrm{L} 4 / 5$ level, lateral lesions in the L5/S1 level, or double-crush lesions.

Table 2. Percentage of cases in the 2 groups reporting symptoms based on the JOABPEQ

\begin{tabular}{|c|c|c|c|c|}
\hline \multicolumn{2}{|c|}{ Questionnaire } & \multirow{2}{*}{$\begin{array}{c}\text { LFS (\%) } \\
84.61\end{array}$} & \multirow{2}{*}{$\begin{array}{c}\text { LSCS (\%) } \\
53.33\end{array}$} & \multirow{2}{*}{$\begin{array}{c}\text { p-value } \\
0.0512\end{array}$} \\
\hline Q 1-1 & Change posture often & & & \\
\hline Q 1-2 & Lie down to rest & 61.53 & 46.66 & 0.3702 \\
\hline Q 1-3 & Lower back aches & 53.84 & 43.33 & 0.5255 \\
\hline Q 1-4 & $\star$ Cannot sleep well & 53.84 & 16.66 & $0.0125^{*}$ \\
\hline Q 2-1 & Require help for tasks & 23.07 & 20.00 & 0.6198 \\
\hline Q 2-2 & Avoid bending forward or kneeling & 46.15 & 43.33 & 0.8641 \\
\hline Q 2-3 & $\star$ Have difficulty standing up from chair & 53.84 & 6.66 & $0.0004 *$ \\
\hline Q 2-4 & $\star$ Have difficulty turning over in bed & 76.92 & 40.00 & $0.0261^{*}$ \\
\hline Q 2-5 & $\star$ Have difficulty putting on socks & 76.92 & 26.66 & $0.0021^{*}$ \\
\hline Q 2-6 & Have difficulty: bending forward, kneeling or stooping & 23.07 & 16.66 & 0.6198 \\
\hline Q 3-1 & Can only walk short distances & 84.61 & 73.33 & 0.4212 \\
\hline Q 3-2 & Stay seated most of the day & 46.15 & 40.00 & 0.7071 \\
\hline Q 3-3 & Go up the stairs more slowly than usual & 92.30 & 83.33 & 0.4353 \\
\hline Q 3-4 & Have difficulty climbing stairs & 30.76 & 23.33 & 0.6077 \\
\hline Q 3-5 & $\star$ Cannot walk more than 15 minutes & 61.53 & 26.66 & $0.0298^{*}$ \\
\hline Q 4-1 & $\star$ Cannot do housework & 38.46 & 0 & $0.0003^{*}$ \\
\hline Q 4-2 & Cannot engage in ordinary activities & 46.15 & 20.00 & 0.0791 \\
\hline Q 4-3 & Work routine is hindered & 61.53 & 36.66 & 0.4296 \\
\hline Q 5-1 & Feel irritated & 30.76 & 40.00 & 0.5652 \\
\hline Q 5-2 & Poor health condition & 76.92 & 60.00 & 0.2849 \\
\hline Q 5-3 & Feel depressed or discouraged & 38.46 & 30.00 & 0.5865 \\
\hline Q 5-4 & Feel exhausted & 46.15 & 26.66 & 0.2104 \\
\hline Q 5-5 & Do not feel happy & 46.15 & 30.00 & 0.3073 \\
\hline Q 5-6 & $\star$ Are not in decent health & 69.23 & 30.00 & $0.0166^{*}$ \\
\hline Q 5-7 & Worry health will get worse & 53.84 & 43.33 & 0.5255 \\
\hline
\end{tabular}

JOABPEQ, Japanese Orthopaedic Association Back Pain Evaluation Questionnaire; LFS, lumbar foraminal stenosis; LSCS, lumbar spinal canal stenosis.

$* \mathrm{p}<0.05$. 

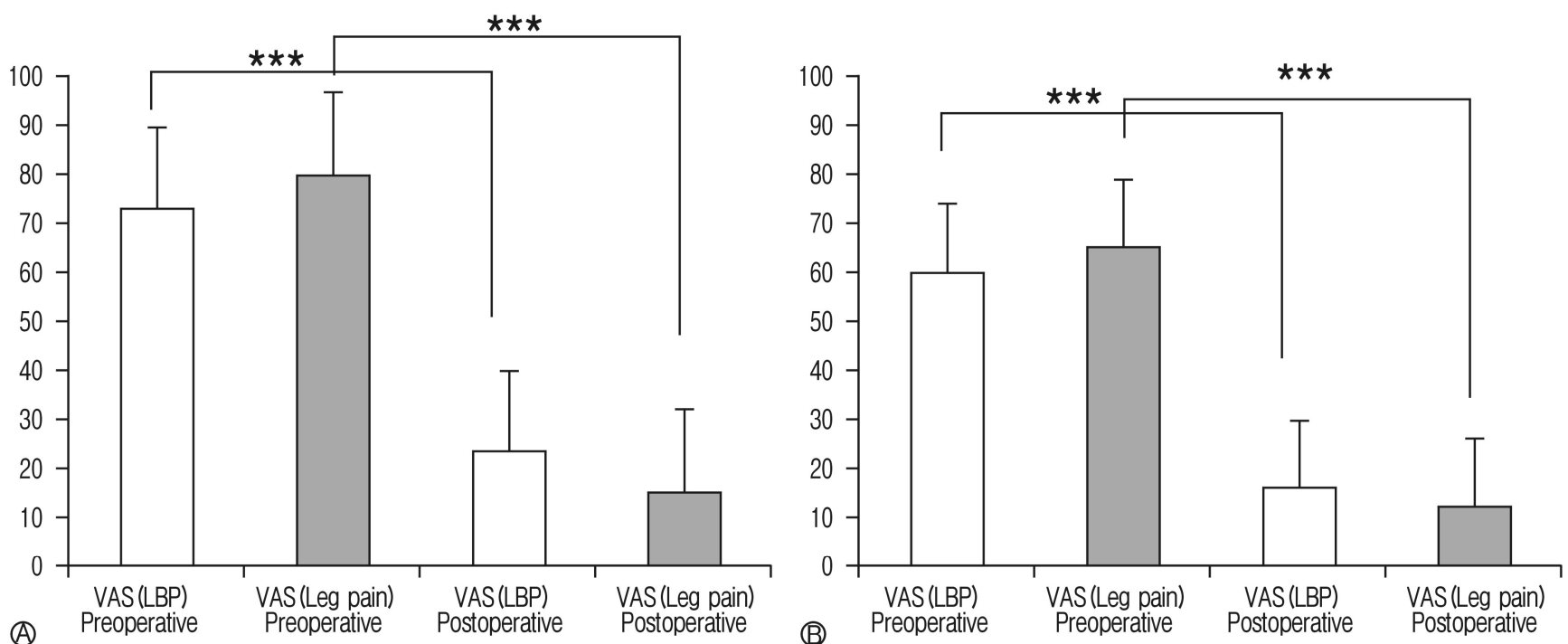

Fig. 3. VAS scores for low back pain (LBP) and leg pain. Both of LBP and leg pain were significantly improved by surgery ( $<<0.001$ ) in LFS patients (A) and LSCS patients (B). LFS, lumbar foraminal stenosis; LSCS, lumbar spinal canal stenosis. $* *$ p $<0.001$.

Electrophysiological tests to measure distal latency of the L5 have been reported. Compared to medial lesions, distal latency is delayed in lateral lesions allowing for a differential diagnosis between the two. However, this is an invasive test and noninvasive diagnostic methods are virtually nonexistent ${ }^{1,99}$.

Due to DRG involvement in patients with symptomatic LFS, they have generally been recognized as demonstrating more severe symptoms than patients with $\operatorname{LSCS}^{3,10,18,19)}$.

In this study we investigated clinical symptoms specific to patients with LFS symptoms. JOA scores were significantly lower in LFS compared to LSCS and lumbar spine dysfunction and social life disturbance in JOABPEQ-measured functionality was also significantly lower in those with LFS.

The RDQ and Oswestry Disability Index are used as specific scales of low-back-pain associated quality of life, while SF-36 and EuroQol are widely used around the world as comprehensive measures of health. JOABPEQ is a patient-based assessment of treatment results that includes both the scientific and psychological aspects ${ }^{4}$. An excel file can be shared from the JOA website, allowing for automatic assessment of individual patient severity.

Statistically significant differences were noted in 7 of the JOABPEQ domains, namely difficulties in: (1) sleeping, (2) standing up from a chair, (3) turning over, (4) putting on socks, (5) walking for 15 minutes, (6) doing household chores, and (7) remaining in decent health.

In previous reports, Yamada et al. ${ }^{19)}$ found that pain when recumbent, pain on sitting, the Bonnet test, and the Freiberg test were specific symptoms of LFS. Watanabe et al ${ }^{18)}$ reported that incidences of Kemp test, intermittent claudication, leg pain in a sitting position, and leg pain at night were high among LFS patients. Baba et al. ${ }^{3)}$ reported that all patients suffered from leg pain caused by nerve root involvement, and the incidence of Kemp sign (84.6\%), intermittent claudication (84.6\%), and leg pain at rest (61.5\%) were all high.

Yamada et al..$^{19)}$ developed a support tool to clinically diagnose symptomatic LFS. They assigned an integer score to each identified risk factor as follows: pain when recumbent, 9 points; positive Freiberg test result, 5 points; positive Bonnet test result, 3 points; and pain on sitting, 3 points. For each patient, all applicable risk score values were added for a total risk score which ranged from 0 to 20 . Receiver operating characteristic (ROC) curve analysis demonstrated cutoff value was 5 points, and the area under the ROC curve was 0.87435 , with a sensitivity of $75.5 \%$, and specificity of $82.3 \%$.

In this study, a high incidence of difficulties were reported in LFS patients such as in sleeping (53.8\%), getting up from a chair (53.8\%), turning over in bed (76.9\%), putting on socks (76.9\%) and intermittent claudication such as resting state pain and inability to walk 15 minutes or more (61.5\%), findings which do not differ from previously published reports. Our study suggests that if resting state pain and intermittent claudication are noted on the JOABPEQ, an established and widely available patient based outcome scale, further diagnosis and treatment should be considered with potential LFS in mind. By using existing and established assessment methods, it may be possible to diagnose LFS easily in a general practice setting.

We acknowledge that our study has several limitations.

First, only a small number of subjects were investigated. Further studies are needed to investigate whether our findings remain valid in a large population. Second, JOABPEQ only assesses lumbar pain and there are no questions related to lower limb pain. Jones et al. ${ }^{11)}$ reported that there was a significant improvement in LBP in patients with LSCS undergoing spinal decompression. In this study, VAS score (leg pain) decreased from LFS: $80 \pm 5.37 \mathrm{~mm}$ and LSCS: $65 \pm 5.25 \mathrm{~mm}$ to LFS: $16 \pm 5.70 \mathrm{~mm}$ $(\mathrm{p}<0.001)$ and LSCS: $13 \pm 3.11 \mathrm{~mm}(\mathrm{p}<0.001)$ after decompressed surgery. VAS score (LBP) decreased from LFS: $73 \pm 5.17 \mathrm{~mm}$ 
and LSCS: $60 \pm 4.67 \mathrm{~mm}$ to LFS: $24 \pm 7.04 \mathrm{~mm}(\mathrm{p}<0.001)$ and LSCS: $16 \pm 3.30 \mathrm{~mm}(\mathrm{p}<0.001)$ after decompressed surgery. Not only leg pain but also LBP significantly improved by decompressed surgery in both group (Fig. 3). There are a number of possible explanations for this phenomenon such as improvement of posture, distressed facet joint, and improved nutrient supply to ischemic nerves ${ }^{11)}$. Radicular LBP has been suggested and further investigation into this issue is necessary. Finally, in this study we looked at spinal stenosis in LSF patients, but no comparisons were made with lumbar disc herniation, so further investigations will be necessary.

\section{CONCLUSION}

We looked into using the JOABPEQ scale to diagnose LFS in symptomatic patients. Compared to LSCS, those with LFS had significantly lower JOA scores and both the lumbar spine dysfunction and social life disturbance scores on the JOABPEQ scale were also significantly lower. JOABPEQ scores showed a significantly higher incidence of difficulties in: sleeping, getting up from a chair, turning over, and putting on socks together such as resting state pain with a higher incidence of intermittent claudication in those with LFS. Results suggest that of the items in the JOABPEQ, if pain during rest or intermittent claudication is noted, LFS should be kept in mind as a cause during subsequent diagnosis and treatment. JOABPEQ may be used as an easy way to differentially diagnose LFS from LSCS.

\section{CONFLICT OF INTEREST}

No potential conflict of interest relevant to this article was reported.

\section{REFERENCES}

1. Ando M, Tamaki T, Kawakami M, Minamide A, Nakagawa Y, Maio K, et al: Electrophysiological diagnosis using sensory nerve action potential for the intraforaminal and extraforaminal L5 nerve root entrapment. Eur Spine J 22:833-839, 2013

2. Aota Y, Niwa T, Yoshikawa K, Fujiwara A, Asada T, Saito T: Magnetic resonance imaging and magnetic resonance myelography in the presurgical diagnosis of lumbar foraminal stenosis. Spine (Phila $\mathrm{Pa}$ 1976) 32:896-903, 2007

3. Baba H, Uchida K, Maezawa Y, Furusawa N, Okumura Y, Imura S: Microsurgical nerve root canal widening without fusion for lumbosacral intervertebral foraminal stenosis: technical notes and early results. Spinal Cord 34:644-650, 1996

4. Burton CV, Kirkaldy-Willis WH, Yong-Hing K, Heithoff KB: Causes of failure of surgery on the lumbar spine. Clin Orthop Relat Res (157):191-199, 1981

5. Eguchi Y, Ohtori S, Orita S, Kamoda H, Arai G, Ishikawa T, et al: Quantitative evaluation and visualization of lumbar foraminal nerve root entrapment by using diffusion tensor imaging: preliminary results. AJNR Am J Neuroradiol 32:1824-1829, 2011

6. Fukui M, Chiba K, Kawakami M, Kikuchi S, Konno S, Miyamoto $\mathrm{M}$, et al: JOA Back Pain Evaluation Questionnaire (JOABPEQ)/JOA Cervical Myelopathy Evaluation Questionnaire (JOACMEQ). The report on the development of revised versions. April 16, 2007. The Subcommittee of the Clinical Outcome Committee of the Japanese Orthopaedic Association on Low Back Pain and Cervical Myelopathy Evaluation. J Orthop Sci 14:348-365, 2009

7. Hasegawa T, An HS, Haughton VM, Nowicki BH: Lumbar for aminal stenosis: critical heights of the intervertebral discs and foramina. A cryomicrotome study in cadavera. J Bone Joint Surg Am 77:32-38, 1995

8. Herron LD: Selective nerve root block in patient selection for lumbar surgery: surgical results. J Spinal Disord 2:75-79, 1989

9. Iwasaki $\mathrm{H}$, Yoshida M, Yamada $\mathrm{H}$, Hashizume $\mathrm{H}$, Minamide $\mathrm{A}$, Nakagawa Y, et al: A new electrophysiological method for the diagnosis of extraforaminal stenosis at L5-s1. Asian Spine J 8:145149,2014

10. Jenis LG, An HS: Spine update. Lumbar foraminal stenosis. Spine (Phila Pa 1976) 25:389-394, 2000

11. Jones AD, Wafai AM, Easterbrook AL: Improvement in low back pain following spinal decompression: observational study of 119 patients. Eur Spine J 23:135-141, 2014

12. Kirkaldy-Willis WH, Wedge JH, Yong-Hing K, Tchang S, de Korompay V, Shannon R: Lumbar spinal nerve lateral entrapment. Clin Orthop Relat Res (169):171-178, 1982

13. Krudy AG: MR myelography using heavily T2-weighted fast spinecho pulse sequences with fat presaturation. AJR Am J Roentgenol 159:1315-1320, 1992

14. Macnab I: Negative disc exploration. An analysis of the causes of nerve-root involvement in sixty-eight patients. J Bone Joint Surg Am 53:891-903, 1971

15. Ozeki N, Aota Y, Uesugi M, Kaneko K, Mihara H, Niimura T, et al: Clinical results of intrapedicular partial pediculectomy for lumbar foraminal stenosis. J Spinal Disord Tech 21:324-327, 2008

16. Vanderlinden RG: Subarticular entrapment of the dorsal root ganglion as a cause of sciatic pain. Spine (Phila Pa 1976) 9:19-22, 1984

17. Watanabe K, Hosoya T, Shiraishi T, Matsumoto M, Chiba K, Toyama Y: Lumbar spinous process-splitting laminectomy for lumbar canal stenosis. Technical note. J Neurosurg Spine 3:405-408, 2005

18. Watanabe K, Yamazaki A, Morita O, Sano A, Katsumi K, Ohashi M: Clinical outcomes of posterior lumbar interbody fusion for lumbar foraminal stenosis: preoperative diagnosis and surgical strategy. J Spinal Disord Tech 24:137-141, 2011

19. Yamada $H$, Oka $H$, Iwasaki $H$, Endo T, Kioka M, Ishimoto $Y$, et al: Development of a support tool for the clinical diagnosis of symptomatic lumbar intra- and/or extra-foraminal stenosis. J Orthop Sci 20:811-817, 2015

20. Yamada H, Terada M, Iwasaki H, Endo T, Okada M, Nakao S, et al: Improved accuracy of diagnosis of lumbar intra and/or extraforaminal stenosis by use of three-dimensional MR imaging: comparison with conventional MR imaging. J Orthop Sci 20:287-294, 2015 